\title{
Women Health Awareness, Acquisitions, and Partnerships to Bring New Era for Contraceptive Drugs.
}

\author{
Ashish Gujrathi \\ Allied Market Research, Viman Nagar Pune Sainik Nagar, Somnath Nagar, Wadgaon Sheri, Pune, Maharashtra, India.
}

Corresponding Author: Ashish Gujrathi, Allied Market Research, Viman Nagar Pune Sainik Nagar, Somnath Nagar, Wadgaon Sheri, Pune, Maharashtra, India.

Received date: September 27, 2021; Accepted date: November 22, 2021; Published date: January 04, 2021

Citation: Ashish G.(2022) Women Health Awareness, Acquisitions, and Partnerships to Bring New Era for Contraceptive Drugs.. J. Obstetrics Gynecology and Reproductive Sciences 6(1) DOI:10.31579/2578-8965/099

Copyright: () 2022, Ashish Gujrathi, This is an open access article distributed under the Creative Commons Attribution License, which permits unrestricted use, distribution, and reproduction in any medium, provided the original work is properly cited.

The spread of COVID-19 and the resulted lockdown imposed across the globe has negatively affected the contraceptive drugs market. Many women across the world found it hard to purchase the pills as contraceptive drug manufacturers were facing challenges due to inconsistent supply chains and the workforce.

However, as the lockdown is getting lifted across the globe, the market is expected to gain traction. Due to the rise in online availability of contraceptive drugs, customers can have easy access to them, which works in favour of the market.

As the growth of the market is visible to many experts and many credible market research reports are expecting a positive future, the market is witnessing some trends such as partnerships, acquisitions, launching new drugs, research and development, and favorable government regulations.

As per the market research report published by Allied Market Research, the global contraceptive drugs market is anticipated to reach $\$ 24,412.22$ million by 2027, manifesting a CAGR of $6.9 \%$ from 2020 to 2027.

Platforms to help drug producers:

As the lockdown has been lifted and online sale of contraceptives is gaining popularity, organizations are launching new techniques and platforms that help drug manufacturers develop cost-efficient and effective drugs.

Agilum Healthcare Intelligence has recently announced the official launch of CRCAtm, which is Comparative Rapid Cycle Analytics® P\&T. It is a new-gen analytics platform developed for drugs manufacturing companies. It comes with robust functions and increases the processing speed.

It helps to check drug manufacturing cost and payment by the payer. It aids hospital pharmacies increase their drug use, improve results, and decline the total cost of medical care treatment.
Favourable government regulations:

Governments in developed countries such as Canada are passing favourable laws to help in boosting women's health. One such regulation was announced recently.

Nextstellis is a new birth control pill launched in the market for Canadian women. What makes this contraceptive pill different from others is that it is made using a plant-based estrogen. While most of the contraceptive pills in the market use ethinylestradiol, which is a synthetic Estrogen, Nextstellis uses estetrol (E4).

As per the claims made by Dr. Brian Hauck of Foothills Medical Centre, the pill does not affect the liver in the way a traditional pill does. The doctor says the research into E4 has been ongoing for years, and it has finally got approval from the Canadian regulatory body.

Canada is one of the earlier countries to provide regulatory approval and make it available in the market. Health Canada approved the drug for public use in March.

Acquisitions in the contraceptive market:

An acquisition is a common trend for a growing market. The contraceptive drugs market is not an exception to it.

A known contraceptive drugs manufacturer HRA Pharma is recently acquire by Perrigo Company plc. HRA is a leading global consumer selfcare company and also one of the fastest-growing over-the-counter ("OTC") companies across the globe.

This acquisition will make Perrigo a global leader in consumer self-care and strengthen its presence in the high-potential European region. It also helps Perrigo to extend its offerings with the help of brands such as a trusted OTC brand Compeed ${ }^{\circledR}$, an OTC emergency contraception brand ellaOne $\AA$, and the OTC scar care brand Mederma ${ }^{\circledR}$. HRA Pharma is an emergency contraception leader in the European region. 